Promoting the uptake of HIV testing among men who have sex with men (MSM): systematic review of effectiveness and cost-effectiveness

Corresponding author:

Dr Theo Lorenc

Department of Social and Environmental Health Research

London School of Hygiene \& Tropical Medicine

15-17 Tavistock Place

London WC1H 9SH

U.K.

Tel: $+44(0) 2079272778$

Fax: +44 (0)20 79272701

E-mail: theo.lorenc@1shtm.ac.uk

Theo Lorenc

Department of Social and Environmental Health Research, London School of Hygiene \& Tropical Medicine, London, UK

Isaac Marrero-Guillamón

Department of Iberian and Latin American Studies, Birkbeck College, London, UK

Peter Aggleton

School of Education and Social Work, University of Sussex, Brighton, UK

Chris Cooper

Centre for Evidence and Policy, King's College London, London, UK

Alexis Llewellyn

Matrix Evidence, London, UK

Angela Lehmann

Matrix Evidence, London, UK

Catriona Lindsay

Matrix Evidence, London, UK

Keywords (MeSH):

AIDS Serodiagnosis/psychology

Health Behavior

HIV Infections/diagnosis

Homosexuality, Male

Systematic Review

Word count: 2,936 


\section{Promoting the uptake of HIV testing among men who have sex with men (MSM): systematic review of effectiveness and cost-effectiveness}

\section{ABSTRACT}

Objectives: What interventions are effective and cost-effective in increasing the uptake of HIV testing among men who have sex with men (MSM)?

Design: Systematic review.

Data sources: AEGIS, ASSIA, BL Direct, BNI, Centre for Reviews and Dissemination, Cochrane Database of Systematic Reviews, CINAHL, Current Contents Connect, EconLit, EMBASE, ERIC, HMIC, Medline, Medline In-Process, NRR, PsychINFO, Scopus, SIGLE, Social Policy and Practice, Web of Science, websites, journal hand-searching, citation chasing and expert recommendations. Review methods: Prospective studies of the effectiveness or cost-effectiveness of interventions (RCT, controlled trial, one-group or any economic analysis) were included if the intervention aimed to increase the uptake of HIV testing among MSM in a high-income (OECD) country. Quality was assessed, and data extracted, using standardised tools. Results were synthesized narratively. Results: Twelve effectiveness studies and one cost-effectiveness study were located, covering a range of intervention types. There is evidence that rapid testing and counselling in community settings (one RCT), and intensive peer counselling (one RCT), can increase uptake of HIV testing among MSM. There are promising results regarding the introduction of opt-out testing in STI clinics (two one-group studies). Findings regarding other interventions, including bundling HIV tests with other tests, peer outreach in community settings, and media campaigns, are inconclusive. 
Conclusions: Findings indicate several promising approaches to increasing HIV testing among MSM. However, there is limited evidence overall, and evidence for the effectiveness of key intervention types (particularly peer outreach and media campaigns) remains lacking. 


\section{Promoting the uptake of HIV testing among men who have sex with men (MSM): systematic review of effectiveness and cost-effectiveness}

There are estimated to be 33,300 MSM living with HIV in the UK, of whom 8,950 are undiagnosed (2008 figures).[1] Strategies to prevent HIV in this population include public health education and community-based awareness-raising about the risks of HIV, and the promotion of safer sex through condom distribution, outreach work and other means. Increasing the uptake of voluntary counselling and testing (VCT) also has a potentially important role to play in reducing the incidence of HIV. Of MSM attending genitourinary medicine (GUM) clinics in 2008, 3.1\% were found to have a previously undiagnosed HIV infection.[1] Promoting HIV testing has the potential to reduce undiagnosed infections, hence improving individual outcomes and reducing transmission. It may also be of value in raising awareness of HIV more broadly, and engaging MSM with sexual health services.

Promoting HIV testing may be of particular value in reducing late diagnoses. Of new HIV diagnoses among MSM in 2008, 43\% had CD4 cell counts below the threshold at which treatment should normally be commenced $\left(<350\right.$ per $\mathrm{mm}^{3}$ within three months of diagnosis), and $20 \%$ had CD4 counts less than 200 per $\mathrm{mm}^{3}$.[1] Late HIV diagnosis is an important predictor of morbidity and mortality. Those who start treatment at a more advanced stage of disease respond less well to treatment, and remain at increased risk of death for many years following treatment initiation.[2]

Rates of HIV testing among MSM vary widely between countries,[3] and between subgroups of MSM within the UK.[4] Rates have varied over time; survey data show 
a steady upward trend in numbers ever tested for HIV in samples of MSM in the UK since 2000.[5] Testing policies also vary internationally, although there is a general trend towards recommending more routine testing.[6] Current UK guidelines recommend that HIV testing should be offered to MSM annually, and more frequently if symptoms indicative of seroconversion or high risk exposure are present;[7] US guidelines recommend annual screening for MSM who themselves or whose sex partners have had more than one sex partner since their most recent HIV test.[8] 'Optout' testing policies in STI clinics are now widely implemented, having been recommended by WHO and UNAIDS in 2004,[9] and by UK guidelines in 2006.[10] Current UK guidelines also recommend opt-out testing in a much wider range of healthcare settings.[7]

We aimed to systematically review the evidence on the effectiveness and costeffectiveness of interventions to increase uptake of HIV testing among MSM. The study presented here is part of a larger review, which also included relevant qualitative evidence, commissioned by NICE (the review protocol has not been published). The full methods and results are available at http://www.nice.org.uk/guidance/index.jsp?action=download\&o=50931. This review includes only interventions which aimed to promote HIV testing, although it is clear that the promotion of HIV testing should form part of an integrated risk-reduction strategy aiming to change a range of sexual behaviours.[11-12]

To our knowledge, no systematic review with this scope has previously been conducted. Two high-quality systematic reviews whose scope partially overlaps with that of the present review were located by our searches. One of these focused on mass 
media interventions to promote HIV testing, but included all populations, not only MSM.[13] The other included HIV health promotion interventions for MSM in general, but did not have a primary focus on HIV testing, and did not include a separate synthesis of evidence relating to interventions promoting HIV testing.[14]

\section{METHODS}

\section{Identification of studies}

We searched the following databases from 1996 to December 2009: AEGIS (AIDSLine and International AIDS Society abstract archives); ASSIA; BL Direct; British Nursing Index; Centre for Reviews and Dissemination databases (including DARE and NHS EED); Cochrane Library (including the Health Technology Assessment database and CENTRAL); CINAHL; Current Contents Connect ; EconLit; EMBASE; ERIC (Education Resources Information Centre); HMIC; Medline; Medline In-Process; National Research Register; PsycINFO; Scopus; SIGLE; Social Policy and Practice; Web of Science (including Social Science Citation Index, Science Citation Index, and Conference Proceedings Citation Index).

Where possible, searches were limited to studies of humans published in English. The Medline search strategy is presented in web-only supplement 1. Full search strategies are available in the complete review report.[15] Searching was conducted simultaneously for this review and the review of qualitative evidence (the strategy was designed to locate both types of evidence, and all references were screened for inclusion in both reviews). 
In addition, we hand-searched nine key journals from January 2005 to December 2009; scanned the citation lists of included studies and those of systematic reviews which met all other criteria for inclusion; and conducted 'forward' citation chasing on included studies using ISI Web of Knowledge. Finally, a call for evidence to experts and stakeholders was issued; all references arising from the call were screened for inclusion.

\section{Inclusion criteria}

The inclusion criteria were as follows:

1) Does the study include men who have sex with men (MSM), or focus on services aimed at MSM?

2) Does the study relate to HIV testing?

3) Was the study conducted in a country which is a current member of the OECD?

4) Was the study published in 1996 or later?

5) Does the study include MSM who are HIV-negative or do not know their HIV status, and are competent to consent to an HIV test?

6) Does the study relate to an intervention that aims to increase the uptake of HIV testing?

7) Is the study a prospective outcome evaluation (randomised or non-randomised controlled trial, or one-group before-and-after study), or a cost-benefit or costeffectiveness analysis, or any other type of economic evaluation? 
For criterion (1), studies which either had a sample more than $50 \%$ of whom were MSM, or presented disaggregated outcome data on the MSM within the sample, or focused on services aimed at MSM, were included. Studies of the general population which did not present outcome data on MSM were excluded. Studies published prior to 1996 were excluded (criterion 4) due to the lesser relevance of data collected before the introduction of effective anti-retroviral treatment for HIV.

For criterion (7), effectiveness studies were included if they either contained a comparison group receiving different interventions (randomised or non-randomised) and/or presented outcome data for both before and after the intervention, or both. Any economic evaluation was included regardless of study design. Systematic reviews were not included; however, systematic reviews meeting criteria (1)-(6) were retained and their lists of included primary studies scanned for inclusion.

A random sample of $10 \%$ of abstracts was screened by two reviewers independently. Inter-rater reliability (Cohen's kappa) was $\kappa=0.627$. Subsequently all abstracts were screened by one reviewer alone. For all references included on abstract, the full text was retrieved and re-screened independently by two reviewers, with disagreements resolved by discussion and reference to a third reviewer if necessary.

\section{Study quality}

Study quality was assessed using a standardised tool.[16] The full quality assessment tool is presented in web-only supplement 2 . Both quality assessment and data extraction were conducted for a sample of $10 \%$ of studies $(\mathrm{N}=2)$ by two reviewers 
independently; subsequently all references were quality-assessed by one reviewer and then checked in detail by a second reviewer. On the basis of this quality assessment, each study was assigned a rating: high $(++)$, medium $(+)$ or low $(-)$. Quality ratings are presented for each study below; studies were not formally weighted by quality in the synthesis.

\section{Data extraction and synthesis}

Data were extracted from the studies using a standardised tool.[16] The list of variables for which data were extracted is presented in web-only supplement 2 . Outcome data were only extracted regarding the uptake of HIV testing and the number of new diagnoses; data on other outcomes (e.g. condom use) were not extracted. Due to the heterogeneity of intervention types, quantitative meta-analysis was not carried out, and studies were synthesized narratively.

\section{RESULTS}

A total of 4,342 unique references were located by our searches (this figure includes studies for the qualitative review as well as those for this review). After screening, a total of 14 study reports on effectiveness and one on cost-effectiveness were retained in the review. Two of these were linked reports presenting data from the same study. Hence, a total of 12 effectiveness studies and one cost-effectiveness study were included. The flow of literature through the review is presented in Figure 1. 
Basic characteristics of the study settings and populations are set out in Table 1. Table 1 also shows the results of the quality assessment process (full details are given in the full report).[15] Table 2 sets out the interventions evaluated and the findings of the studies. We have divided the interventions into four categories:

1) offering different types of test or testing protocols in outreach settings;[17-19]

2) peer education or recruitment programmes, including intensive residential programmes[20] and community-based programmes;[21-23]

3) community-based media campaigns[24-25] and web-based educational interventions;[26] and

4) changes to the way in which services are delivered in STI clinics, including the introduction of opting-out schemes[27-28] and the implementation of guidelines recommending regular screening.[29]

Broadly, these four categories can be grouped as service delivery interventions ( 1 and 4) and community-level interventions (2 and 3). 
Table 1. Summary of included studies: population characteristics

\begin{tabular}{|c|c|c|c|c|c|c|c|c|}
\hline $\begin{array}{l}\text { Study } \\
\text { reference }\end{array}$ & $\begin{array}{l}\text { Study } \\
\text { type }\end{array}$ & $\begin{array}{l}\text { Quality } \\
\text { score }\end{array}$ & $\begin{array}{l}\text { Population } \\
\text { targeted }\end{array}$ & Country & $\begin{array}{l}\text { Sampling and } \\
\text { recruitment }\end{array}$ & $\begin{array}{l}\text { Sample } \\
\text { size }\end{array}$ & $\begin{array}{l}\text { Baseline } \\
\text { demographics }\end{array}$ & $\begin{array}{l}\text { Baseline HIV } \\
\text { testing experience }\end{array}$ \\
\hline \multicolumn{9}{|c|}{ Type of test / testing protocol } \\
\hline $\begin{array}{l}\text { Spielberg et al. } \\
2005[18]\end{array}$ & $\mathrm{RCT}$ & ++ & $\begin{array}{l}\text { MSM and } \\
\text { injecting drug } \\
\text { users (only } \\
\text { MSM findings } \\
\text { reported here) }\end{array}$ & USA & $\begin{array}{l}\text { All men using } \\
\text { bathhouse } \\
\text { approached to } \\
\text { participate }\end{array}$ & $\begin{array}{l}3,140 \\
\text { (partici } \\
\text { pated) } \\
561 \\
\text { (agreed } \\
\text { to test) }\end{array}$ & $\begin{array}{l}\text { Age: } 22 \%<30 \\
\text { Ethnicity: } 80 \% \text { white } \\
\text { (estimated, for eligible } \\
\text { population) }\end{array}$ & $\begin{array}{l}6 \% \text { never (of } \\
\text { eligible population; } \\
\text { those tested }<3 \\
\text { months ago were } \\
\text { excluded) }\end{array}$ \\
\hline $\begin{array}{l}\text { Galvan et al. } \\
2006[17]\end{array}$ & nRCT & + & Latino MSM & USA & $\begin{array}{l}\text { Randomly } \\
\text { sampled from } \\
\text { men entering bars } \\
\text { popular with } \\
\text { Latino MSM }\end{array}$ & 394 & $\begin{array}{l}\text { Ethnicity: All Latino. } \\
\text { Other details NR for } \\
\text { eligible population } \\
\text { (only for those men } \\
\text { agreeing to test) }\end{array}$ & NR \\
\hline $\begin{array}{l}\text { Spielberg et al. } \\
2000[19]\end{array}$ & $\mathrm{RCT}$ & ++ & $\begin{array}{l}\text { MSM, injecting } \\
\text { drug users and } \\
\text { women at } \\
\text { heterosexual } \\
\text { risk (only MSM } \\
\text { findings } \\
\text { reported here) }\end{array}$ & USA & $\begin{array}{l}\text { From the HIV } \\
\text { Network for } \\
\text { Prevention Trials } \\
\text { Vaccine } \\
\text { Preparedness } \\
\text { Study (VPS) } \\
\text { Cohort }\end{array}$ & 140 & NR for MSM group & NR \\
\hline \multicolumn{9}{|c|}{ Peer education and recruitment } \\
\hline $\begin{array}{l}\text { Flowers et al. } \\
2002[21,30]\end{array}$ & $\mathrm{nRCT}$ & + & MSM & Scotland & $\begin{array}{l}\text { All men entering } \\
\text { one of } 10 \text { gay } \\
\text { bars in given time }\end{array}$ & $\begin{array}{l}4,774 \\
\text { (total) }\end{array}$ & $\begin{array}{l}\text { Age: mean } 31.7 \text { years. } \\
\text { SES: } 87 \% \text { social class } \\
\text { I-III. }\end{array}$ & $49.3 \%$ never \\
\hline
\end{tabular}




\begin{tabular}{|c|c|c|c|c|c|c|c|c|}
\hline & & & & & $\begin{array}{l}\text { period were } \\
\text { approached }\end{array}$ & & $\begin{array}{l}\text { Education: } 40 \% \text { at least } \\
\text { degree level. }\end{array}$ & \\
\hline $\begin{array}{l}\text { Elford et al. } \\
2000[22,31]\end{array}$ & $\mathrm{nRCT}$ & - & MSM & England & $\begin{array}{l}\text { Peer educators } \\
\text { recruited from } \\
\text { gyms with } \\
\text { predominantly } \\
\text { gay clientele by } \\
\text { gym managers }\end{array}$ & $\begin{array}{l}5,656 \\
\text { (total) }\end{array}$ & $\begin{array}{l}\text { Age: median } 33 \text { years. } \\
\text { Ethnicity: } 89 \% \text { white. } \\
\text { Employment: } 88 \% \\
\text { employed. }\end{array}$ & $\begin{array}{l}27 \% \text { never, } 10 \%<3 \\
\text { months ago, } 18 \% \\
4-12 \text { months ago, } \\
45 \%>1 \text { year ago }\end{array}$ \\
\hline $\begin{array}{l}\text { Wilton et al. } \\
2009[20]\end{array}$ & $\mathrm{RCT}$ & ++ & Black MSM & USA & $\begin{array}{l}\text { Outreach, } \\
\text { referrals from } \\
\text { service users and } \\
\text { gatekeepers, } \\
\text { advertising }\end{array}$ & 338 & $\begin{array}{l}\text { Age: mean } 29.6 \text { years. } \\
\text { Ethnicity: all self- } \\
\text { identified as Black. } \\
\text { SES: } 46.4 \% \text { income } \\
<\$ 20,000 \text { p.a. } \\
\text { Education: } 29.9 \% \\
\text { college degree. } \\
\text { Sexual orientation: } \\
78.1 \% \text { gay / } \\
\text { homosexual; } 18.3 \% \\
\text { bisexual. }\end{array}$ & $\begin{array}{l}96.1 \% \text { ever, } 41.4 \% \\
<3 \text { months ago }\end{array}$ \\
\hline $\begin{array}{l}\text { Golden et al. } \\
\text { 2006[23] }\end{array}$ & $\begin{array}{l}\text { Economic } \\
\text { analysis } \\
\text { (non- } \\
\text { comparati } \\
\text { ve data) }\end{array}$ & - & MSM & USA & $\begin{array}{l}\text { Through health } \\
\text { services, } \\
\text { advertising, } \\
\text { outreach }\end{array}$ & $\begin{array}{l}283 \\
\text { (recruit } \\
\text { ers) }+ \\
498 \\
\text { (peers) }\end{array}$ & Unclear & Unclear \\
\hline \multicolumn{9}{|c|}{ Media and web-based campaigns } \\
\hline $\begin{array}{l}\text { Guy et al. } \\
2009[24]\end{array}$ & $\begin{array}{l}\text { Before- } \\
\text { and-after }\end{array}$ & - & MSM & Australia & $\begin{array}{l}\text { Participants in } \\
\text { Melbourne Gay } \\
\text { Community } \\
\text { Periodic Survey }\end{array}$ & $\begin{array}{l}4,988 \\
\text { (total) }\end{array}$ & NR for this sample & $\begin{array}{l}60.3 \%<12 \text { months } \\
\text { ago }\end{array}$ \\
\hline
\end{tabular}




\begin{tabular}{|c|c|c|c|c|c|c|c|c|}
\hline $\begin{array}{l}\text { McOwan et al. } \\
2002[25]\end{array}$ & $\begin{array}{l}\text { Before- } \\
\text { and-after }\end{array}$ & - & $\begin{array}{l}\text { MSM, } \\
\text { particularly } \\
\text { black and } \\
\text { southern } \\
\text { European }\end{array}$ & England & Record review & $\begin{array}{l}357 \\
\text { (total) }\end{array}$ & $\begin{array}{l}\text { Age: } 9 \% \text { under } 25 . \\
\text { Ethnicity: } 5 \% \text { 'south } \\
\text { European' origin; } 3 \% \\
\text { Black origin. } \\
\text { (of those seeking HIV } \\
\text { test at targeted clinic) }\end{array}$ & NR \\
\hline $\begin{array}{l}\text { Chiasson et al. } \\
2009[26]\end{array}$ & $\begin{array}{l}\text { Before- } \\
\text { and-after }\end{array}$ & + & MSM & USA & $\begin{array}{l}\text { Recruited through } \\
\text { a banner advert } \\
\text { on a gay sexual } \\
\text { meeting site }\end{array}$ & $\begin{array}{l}3,052 \\
\text { (consen } \\
\text { ted); } \\
1,463 \\
\text { (receive } \\
\mathrm{d} \\
\text { interve } \\
\text { ntion) }\end{array}$ & $\begin{array}{l}\text { Age: } 22 \% 18-29,36 \% \\
30-39,42 \% \geq 40 \text {. } \\
\text { Ethnicity: } 72 \% \text { White. } \\
\text { Education: } 51 \% \text { college } \\
\text { degree. } \\
\text { Sexual orientation: } 91 \% \\
\text { homosexual, } 9 \% \\
\text { bisexual. }\end{array}$ & $6 \%$ never \\
\hline \multicolumn{9}{|c|}{ STI clinic service delivery } \\
\hline $\begin{array}{l}\text { Dukers- } \\
\text { Muijrers et al. } \\
2009[27]\end{array}$ & $\begin{array}{l}\text { Before- } \\
\text { and-after }\end{array}$ & - & $\begin{array}{l}\text { General } \\
\text { population (only } \\
\text { MSM findings } \\
\text { reported here) }\end{array}$ & $\begin{array}{l}\text { Nether- } \\
\text { lands }\end{array}$ & Record review & $\begin{array}{l}1,127 \\
\text { (total) }\end{array}$ & NR for MSM & NR \\
\hline $\begin{array}{l}\text { Heijman et al. } \\
2009[28]\end{array}$ & $\begin{array}{l}\text { Before- } \\
\text { and-after }\end{array}$ & + & $\begin{array}{l}\text { General } \\
\text { population (only } \\
\text { MSM findings } \\
\text { reported here) }\end{array}$ & $\begin{array}{l}\text { Nether- } \\
\text { lands }\end{array}$ & Record review & $\begin{array}{l}8,047 \\
\text { (total) }\end{array}$ & $\begin{array}{l}\text { Age: } 29 \% \leq 29,34 \% \\
30-39,25 \% 40-49,12 \% \\
\geq 50 . \\
\text { Nationality: } 72 \% \text { Dutch }\end{array}$ & $16 \%$ never \\
\hline $\begin{array}{l}\text { Ryder et al. } \\
2005[29]\end{array}$ & $\begin{array}{l}\text { Before- } \\
\text { and-after }\end{array}$ & - & MSM & Australia & $\begin{array}{l}\text { Record review } \\
\text { (random sample) }\end{array}$ & 569 & Age: median 33 years & NR \\
\hline
\end{tabular}


Table 2. Summary of included studies: interventions and outcomes

\begin{tabular}{|c|c|c|c|c|c|}
\hline $\begin{array}{l}\text { Study } \\
\text { reference }\end{array}$ & Intervention and comparison & $\begin{array}{l}\text { Follow-up } \\
\text { (measured } \\
\text { from start of } \\
\text { intervention } \\
\text { delivery) }\end{array}$ & HIV testing outcome & HIV testing findings & $\begin{array}{l}\text { New } \\
\text { diagnoses: } \\
\mathrm{N}(\% \text { of } \\
\text { completed } \\
\text { tests) }\end{array}$ \\
\hline \multicolumn{6}{|c|}{ Type of test / testing protocol } \\
\hline $\begin{array}{l}\text { Spielberg et } \\
\text { al. } 2005[18]\end{array}$ & $\begin{array}{l}\text { Four testing protocols offered in gay } \\
\text { bathhouses. (1) Traditional serum testing and } \\
\text { a return visit to receive results, with standard } \\
\text { face-to-face counselling before testing; (2) } \\
\text { rapid serum testing with same-day test results } \\
\text { and single-session counselling; (3) oral fluid } \\
\text { testing with standard counselling; (4) } \\
\text { traditional serum testing with the choice of } \\
\text { pre-test written materials or standard } \\
\text { counselling. }\end{array}$ & Immediate & $\begin{array}{l}\text { Acceptance of testing; } \\
\text { completion of testing; } \\
\text { receipt of test results }\end{array}$ & $\begin{array}{l}\text { Acceptance. (1): } 15.8 \% ;(2): \\
21.2 \% * ;(3): 22.8 \% * ;(4): 13.6 \% \\
\text { Completion. (1): } 12.4 \% ;(2): \\
\text { 16.2\%*; (3): } 17.3 \% * ;(4): 11.2 \% \\
\text { Receipt. (1): } 9.1 \% ;(2): 16.0 \% * ; \\
\text { (3): } 12.5 \% * ;(4): 8.4 \% \\
\text { [significance tests by comparison } \\
\text { with group (1)] }\end{array}$ & $\begin{array}{l}(1 \text { and } 4): \\
13(6.1 \%) \\
(2 \text { and } 3): 2 \\
(0.9 \%)\end{array}$ \\
\hline $\begin{array}{l}\text { Galvan et } \\
\text { al. 2006[17] }\end{array}$ & $\begin{array}{l}\text { HIV tests bundled with other tests (for other } \\
\text { STIs, alcohol and drug dependence and } \\
\text { depression) offered in bars. Comparison: HIV } \\
\text { tests alone. }\end{array}$ & Immediate & Acceptance of testing & $\begin{array}{l}\text { I (bundled): } 10.2 \% \\
\text { C (HIV alone): } 8.9 \%\end{array}$ & $\begin{array}{l}\text { I (bundled): } \\
5(3.4 \%) \\
\text { C (HIV } \\
\text { alone): } 10 \\
(5.1 \%)\end{array}$ \\
\hline $\begin{array}{l}\text { Spielberg et } \\
\text { al. 2000[19] }\end{array}$ & $\begin{array}{l}\text { Two types of HIV tests for home testing: (1) } \\
\text { dried blood spot home collection, } 3 \text { cycles, } \\
\text { bimonthly. (2) oral fluid home collection, } 3 \\
\text { cycles, bimonthly. }\end{array}$ & 20 weeks & $\begin{array}{l}\text { Adherence to home } \\
\text { specimen collection } \\
\text { schedule }\end{array}$ & $\begin{array}{l}\text { (1) } 92 \% \text {; (2) } 99 \% \text { [not tested for } \\
\text { significance by study authors] }\end{array}$ & $\mathrm{NR}$ \\
\hline \multicolumn{6}{|c|}{ Peer education and recruitment } \\
\hline Flowers et & Risk-reduction education in bars delivered by & 15 months & Self-reported ever & I: $47.0 \%$ baseline, $47.9 \%$ at 15 & NR \\
\hline
\end{tabular}




\begin{tabular}{|c|c|c|c|c|c|}
\hline $\begin{array}{l}\text { Study } \\
\text { reference }\end{array}$ & Intervention and comparison & $\begin{array}{l}\text { Follow-up } \\
\text { (measured } \\
\text { from start of } \\
\text { intervention } \\
\text { delivery) }\end{array}$ & HIV testing outcome & HIV testing findings & $\begin{array}{l}\text { New } \\
\text { diagnoses: } \\
\mathrm{N}(\% \text { of } \\
\text { completed } \\
\text { tests) }\end{array}$ \\
\hline $\begin{array}{l}\text { al. } 2002[21, \\
30]\end{array}$ & $\begin{array}{l}\text { peer educators over } 9 \text { months. Peer educators } \\
\text { received } 2 \text { days of training and support } \\
\text { throughout the intervention. Training involved } \\
\text { communication skills, role play and message } \\
\text { delivery. Comparison: no intervention. }\end{array}$ & & testing & $\begin{array}{l}\text { months. } \\
\text { C: } 55.1 \% \text { baseline, } 52.8 \% \text { at } 15 \\
\text { months. }\end{array}$ & \\
\hline $\begin{array}{l}\text { Elford et al. } \\
2000[22, \\
31]\end{array}$ & $\begin{array}{l}\text { Peer educators were identified on the basis of } \\
\text { social connections and communication skills. } \\
\text { They received training and were asked to talk } \\
\text { to at least } 20 \text { gay men over the next } 5 \text { months } \\
\text { about risk reduction. Comparison: no } \\
\text { intervention. }\end{array}$ & 18 months & $\begin{array}{l}\text { Self-reported ever } \\
\text { testing }\end{array}$ & $\begin{array}{l}\text { I: } 72 \% \text { baseline, } 78 \% \text { at } 18 \\
\text { months. } \\
\text { C: } 78 \% \text { baseline, } 87 \% \text { at } 18 \\
\text { months. }\end{array}$ & NR \\
\hline $\begin{array}{l}\text { Wilton et } \\
\text { al. 2009[20] }\end{array}$ & $\begin{array}{l}\text { Intensive 3-day residential risk-reduction } \\
\text { intervention led by trained Black MSM peers. } \\
\text { The intervention was conducted in a small } \\
\text { group and focused on relationships, HIV risk, } \\
\text { behaviour change, racism and homophobia. } \\
\text { Comparison: waiting list. }\end{array}$ & 6 months & $\begin{array}{l}\text { Self-reported HIV } \\
\text { testing in last } 3 \\
\text { months }\end{array}$ & $\begin{array}{l}\text { I: } 38.4 \% \text { baseline, } 52.0 \% \text { at } 3 \\
\text { months, } 54.8 \% \text { at } 6 \text { months } \\
\text { C: } 44.3 \% \text { baseline, } 46.3 \% \text { at } 3 \\
\text { months, } 43.3 \% \text { at } 6 \text { months } *\end{array}$ & $\begin{array}{l}\text { I: } 4(5.8 \%) \\
\text { C: } 4(6.9 \%)\end{array}$ \\
\hline $\begin{array}{l}\text { Golden et } \\
\text { al. 2006[23] }\end{array}$ & $\begin{array}{l}\text { Peer recruitment programme. Recruiters were } \\
\text { identified from service users and through } \\
\text { advertisements and outreach. They received } \\
40 \text { minutes of training and were offered } \\
\text { US } \$ 20 \text { for each peer recruited (peers also } \\
\text { received } \$ 20 \text { if tested). Peers were tested for } \\
\text { HIV, hepatitis A, B and C, and syphilis. }\end{array}$ & 29 months & $\begin{array}{l}\text { Cost per newly- } \\
\text { identified HIV- } \\
\text { infected person; cost } \\
\text { per newly-identified } \\
\text { person who received } \\
\text { test results }\end{array}$ & $\begin{array}{l}\text { Per new case: US } \$ 4929 \text {. Per new } \\
\text { case who received test results: } \\
\text { US } \$ 5377\end{array}$ & $22(5.0 \%)$ \\
\hline
\end{tabular}




\begin{tabular}{|c|c|c|c|c|c|}
\hline $\begin{array}{l}\text { Study } \\
\text { reference }\end{array}$ & Intervention and comparison & $\begin{array}{l}\text { Follow-up } \\
\text { (measured } \\
\text { from start of } \\
\text { intervention } \\
\text { delivery) }\end{array}$ & HIV testing outcome & HIV testing findings & $\begin{array}{l}\text { New } \\
\text { diagnoses: } \\
\text { N (\% of } \\
\text { completed } \\
\text { tests) }\end{array}$ \\
\hline \multicolumn{6}{|c|}{ Media and web-based campaigns } \\
\hline $\begin{array}{l}\text { Guy et al. } \\
2009[24]\end{array}$ & $\begin{array}{l}\text { Multi-component social marketing campaign, } \\
\text { including display advertising, print and radio } \\
\text { advertisements, and a website. Targeted } \\
\text { messages were aimed at young MSM, non- } \\
\text { gay-community-attached MSM, and culturally } \\
\text { and linguistically diverse MSM. }\end{array}$ & 2 years & $\begin{array}{l}\text { Self-reported testing } \\
\text { in last } 12 \text { months }\end{array}$ & $\begin{array}{l}60.3 \% \text { baseline, } 61.4 \% \text { at } 1 \text { year, } \\
61.9 \% \text { at } 2 \text { years }\end{array}$ & NR \\
\hline $\begin{array}{l}\text { McOwan et } \\
\text { al. 2002[25] }\end{array}$ & $\begin{array}{l}\text { Print media promoting testing at a specific } \\
\text { venue, distributed regularly to gay venues by } \\
\text { community outreach teams. }\end{array}$ & 12 weeks & $\begin{array}{l}\text { Number of tests } \\
\text { carried out at targeted } \\
\text { clinic }\end{array}$ & $\begin{array}{l}\mathrm{N}=65 \text { over } 12 \text { weeks prior to } \\
\text { intervention, } \mathrm{N}=292 \text { over } 12 \text {-week } \\
\text { intervention period * }\end{array}$ & NR \\
\hline $\begin{array}{l}\text { Chiasson et } \\
\text { al. 2009[26] }\end{array}$ & $\begin{array}{l}\text { Educational video (viewed online) designed to } \\
\text { promote critical thinking about HIV risk }\end{array}$ & 3 months & $\begin{array}{l}\text { Self-reported testing } \\
\text { in last } 3 \text { months }\end{array}$ & $26 \%$ baseline, $44 \%$ at 3 months $*$ & $17(14.2 \%)$ \\
\hline \multicolumn{6}{|c|}{ STI clinic service delivery } \\
\hline $\begin{array}{l}\text { Dukers- } \\
\text { Muijrers et } \\
\text { al. 2009[27] }\end{array}$ & Opt-out HIV testing policy in STI clinic & 3 years & Refusal of HIV test & $\begin{array}{l}16 \% \text { at baseline; } 10 \% \\
\text { immediately after intervention; } \\
6 \% \text { in years } 1-2 ; 2.1 \% \text { by end of } \\
\text { year } 3 *\end{array}$ & $\begin{array}{l}(2.8 \%) \\
\text { across study } \\
\text { period: N } \\
\text { NR }\end{array}$ \\
\hline $\begin{array}{l}\text { Heijman et } \\
\text { al. 2009[28] }\end{array}$ & Opt-out HIV testing policy in STI clinic & 11 months & Uptake of HIV test & $\begin{array}{l}62 \% \text { at baseline, } 88 \% \\
\text { immediately after intervention, } \\
93 \% \text { at } 11 \text { months [not tested for } \\
\text { significance by study authors] }\end{array}$ & $\begin{array}{l}95(3.7 \%) \\
\text { baseline; } \\
117(3.4 \%) \\
\text { after } \\
\text { intervention }\end{array}$ \\
\hline Ryder et al. & Implementation of guidelines in STI clinic & 1 year & Proportion of MSM & $73 \%$ baseline, $88 \%$ at follow up & NR \\
\hline
\end{tabular}




\begin{tabular}{|lllll|}
$\begin{array}{l}\text { Study } \\
\text { reference }\end{array}$ & Intervention and comparison & $\begin{array}{l}\text { Follow-up } \\
\text { (measured } \\
\text { from start of } \\
\text { intervention } \\
\text { delivery) }\end{array}$ & HIV testing outcome & HIV testing findings \\
\hline 2005[29] & $\begin{array}{l}\text { recommending at least annual screening of } \\
\text { MSM for HIV and other STIs }\end{array}$ & $\begin{array}{l}\text { (approx.) } \\
\text { New }\end{array}$ & $\begin{array}{l}\text { attendees testing for } \\
\text { Niagnoses: } \\
\text { completed } \\
\text { tests) }\end{array}$ \\
\hline
\end{tabular}

*Significant at $\mathrm{p}=0.05$ according to study authors' analyses 


\section{Type of test and testing protocol}

One study from the USA, published in 2006, compared the uptake of HIV tests included with a package of other tests (for other STIs, alcohol and drug dependence, and depression) offered in outreach settings (bars) with that of HIV tests alone.[17] This study found no significant difference in uptake between the two groups. However, there were some promising but non-significant trends among high-risk subgroups towards greater acceptance of the packaged tests compared to the HIV test alone.

One study, also from the USA and conducted in 1999-2000, compared traditional serum testing and counselling with rapid serum testing and oral fluid testing offered in a gay bathhouse.[18] This study found that both rapid serum testing and oral fluid testing were significantly more likely to be accepted than traditional testing. This study provides evidence that rapid testing modalities can increase uptake in an outreach setting compared to traditional testing.

A further US study, conducted in 1996-97, compared oral fluid and blood spot home testing kits, finding high levels of adherence to both types of test.[19]

\section{Peer education and recruitment}

Two studies from the UK, both from the late 1990s (1996-99), used non-randomised designs to investigate the effectiveness of peer-led community-based risk-reduction campaigns.[21-22] Both of these studies found such campaigns not to be effective in 
increasing HIV testing among a community sample of MSM. However, there are limitations in the design and reporting of both these studies (see Discussion), so they arguably do not provide conclusive evidence for the ineffectiveness of peer-led strategies.

One RCT conducted in 2005-07 evaluated an intensive weekend residential programme for Black MSM in the USA led by trained MSM peers.[20] This study found a small but significant improvement in HIV testing rates among participants at six-month follow-up.

One economic analysis, conducted in 2002-05, looked at a peer recruitment programme to increase rates of HIV testing, finding a cost per new case of HIV identified of US\$4,929.[23] According to the study authors, this compares favourably with costs per case for other interventions, although the analysis presented falls considerably short of a full cost-effectiveness analysis.

\section{Media and web-based campaigns}

Two non-comparative studies in Australia and England investigated communitybased media campaigns to promote HIV testing among MSM, including components such as display advertising and websites and print media distributed to gay venues and organisations.[24-25] One of these studies, conducted in 2004-06, found no increase in the numbers of MSM in the targeted location reporting an HIV test in the previous year.[24] The other, conducted in 1999-2000, found a substantial increase in HIV tests conducted in the targeted clinic.[25] However, there are flaws in the design 
of both these studies (see Discussion). Overall, the evidence for media campaigns must be regarded as inconclusive.

One further non-comparative study in the USA, conducted in 2005-06, evaluated an educational video which was accessed through a banner advert on a gay sexual meeting website.[26] This study found a significant increase in HIV testing at 3month follow-up (and a substantial number of new diagnoses were made as a result). This finding is promising, although since the study is non-comparative, it does not permit strong conclusions to be drawn.

\section{STI clinic service delivery}

Two studies from the mid-2000s (2003-07), both non-comparative, investigated the introduction of 'opt-out' policies in STI clinics in the Netherlands, such that all clients attending the clinic received an HIV test unless they requested not to have one.[2728] Both these studies found substantial increases in HIV tests among MSM after the introduction of the opt-out policy. A finding of concern in both these studies was that certain groups, particularly older MSM and MSM with potentially STI-related symptoms, were more likely to opt out of testing after the introduction of the policy.

One study from 2000-02, also non-comparative, found that the implementation of guidelines promoting regular HIV testing in an STI clinic resulted in an increase in the proportion of MSM being tested, although it is unclear if the increase reached significance.[29] 


\section{DISCUSSION}

This review indicates that several promising strategies are available to increase the uptake of HIV testing among MSM. Opt-out policies in STI clinics can increase testing rates, although there remain concerns about high rates of refusal among certain high-risk subgroups (as well as about those MSM who do not attend STI clinics). Offering rapid testing in outreach settings can also increase the uptake of testing relative to traditional testing methods.

However, there is limited evidence for the effectiveness of community-level strategies. One study shows that a holistic group intervention, led by trained peers, can increase testing among a minority ethnic, socio-economically disadvantaged group of MSM. While this is a promising result, such interventions are resourceintensive and may be challenging to implement. Nonetheless, further research on such strategies would be valuable. There are also promising findings, warranting further research, regarding a Web-based intervention.

Evidence regarding media campaigns and peer-led outreach strategies is inconclusive. Methodologically, evaluations of existing programmes would benefit from measuring individual-level outcomes among men exposed to the intervention, as well as population-level outcomes, and from indexing outcome measures to the timeframe of the intervention (i.e. recent testing rather than lifetime testing). With regard to intervention content, more intensive programmes, sustained over longer timeframes and reaching a larger proportion of the population, are likely to have more impact, as studies of media campaigns in other fields have found.[32] Using formative research, 
or the published qualitative literature, could also be of value in targeting the content of such interventions.

Several limitations in the evidence should be noted. The findings show that, even where interventions succeeded in increasing uptake, numbers of new diagnoses were generally modest. Baseline rates of recent HIV testing in the targeted populations were often high: for example, Spielberg and colleagues' study excluded 35\% of potential participants because they had already had an HIV test within the last three months.[18] In addition, most studies recruited primarily from gay venues and/or MSM-oriented services or organisations, and were unlikely to include less gayidentified MSM. These points raise the question as to whether evaluated interventions reach those MSM who are most at risk of having undiagnosed HIV.

Other limitations relate to the methods of the primary studies, particularly those evaluating community-level interventions. First, several studies used non-comparative designs and were conducted over several years. This makes it difficult to distinguish the effects of the intervention from longer-term upward trends in testing behaviour. Second, several studies measured outcomes at a population level (i.e. different individuals were sampled at pre- and post-test), meaning that any measurement of effectiveness may be diluted by changes in the population. This is particularly an issue when the reach of interventions is limited: two studies found that relatively few of the targeted populations could recall direct contact with the intervention at post-test $(29 \%[21]$ and $3 \%[22])$. A third limitation relates to the measurement of outcomes: the use of lifetime HIV testing rather than recent testing, [21-22] or the total number of 
HIV tests conducted by specific services,[25] may not provide an accurate picture of changes in testing behaviour.

There are also some limitations in the review itself, primarily because of its intentionally restricted scope. This review did not consider evidence relating to HIV testing in populations other than MSM, although this evidence is potentially relevant; for example, there is evidence that mass media interventions are effective in the general population.[13] It also did not consider the considerable evidence on other risk-reduction strategies among MSM. This evidence would be valuable in providing the context needed to understand the longer-term impacts of successful interventions to promote HIV testing among MSM. For example, the role of testing in serosorting strategies, which remain prevalent among some groups of MSM and may involve substantial risk of infection,[33-34] suggests that the relation between the uptake of testing, infection rates, and health status, is complex and unpredictable. Hence, interventions to promote testing, in isolation, may not have a substantial impact on rates of infection. Such interventions are more likely to be effective as part of an integrated programme for reducing HIV infections and promoting access to antiretroviral treatment among MSM.

Key messages:

* Interventions to promote the uptake of HIV testing among men who have sex with men have the potential to reduce HIV risk and late diagnoses

* Opt-out testing policies, and offering rapid testing, can increase the uptake of HIV testing among MSM 
* Evidence concerning the effectiveness of community-level strategies, such as media campaigns or peer education and recruitment, is inconclusive

* Strategies to promote testing should be seen in the broader context of a comprehensive approach to HIV prevention 
Acknowledgements:

This review was funded by the National Institute for Health and Clinical Excellence (NICE). The authors would like to thank the NICE project team (Chris Carmona, Catherine Swann, Daniel Tuvey and Linda Sheppard) for their invaluable support and guidance throughout the review. The views and opinions expressed are those of the authors alone and do not reflect the official opinion of NICE, or any guidance issued by NICE.

Contributions:

TL led the design and conduct of the review. Literature searches were conceived and executed by CC. Screening was carried out by TL, IMG, AL (Alexis Llewellyn), AAL (Angela Lehmann), and CL. Quality assessment and data extraction was carried out by TL and IMG. Data analysis and interpretation were carried out by TL, IMG and PA. TL wrote the first draft of the paper with input from IMG and PA. 
Copyright licence statement

The Corresponding Author has the right to grant on behalf of all authors and does grant on behalf of all authors, an exclusive licence on a worldwide basis to the BMJ Group and co-owners or contracting owning societies (where published by the BMJ Group on their behalf), and its Licensees to permit this article (if accepted) to be published in Sexually Transmitted Infections and any other BMJ Group products and to exploit all subsidiary rights, as set out in our licence. 


\section{References}

1 Health Protection Agency. HIV in the United Kingdom: 2009 report. London: HPA 2009.

2 May M, Sterne JA, Sabin C, et al. Prognosis of HIV-1 infected patients up to 5 years after initiation of HAART: collaborative analysis of prospective studies. AIDS 2007;21:1185-1197.

3 Sullivan PS, Hamouda O, Delpech V, et al. Reemergence of the HIV epidemic among men who have sex with men in North America, Western Europe, and Australia, 1996-2005. Ann Epidemiol 2009;19:423-31.

4 Hickson F, Bourne A, Weatherburn P, et al. Tactical dangers: Findings from the Gay Men's Sex Survey 2008. London: Sigma Research 2010.

5 Hickson F, Weatherburn P, Reid D et al. Testing targets: Findings from the Gay Men's Sex Survey 2007. London: Sigma Research 2009.

6 Mounier-Jack S, Nielsen S, Coker R. HIV testing strategies across European countries. HIV Med 2008;9 suppl. 2:13-9.

7 British HIV Association (BHIVA), British Association of Sexual Health and HIV (BASHH), and British Infection Society (BIS). UK National Guidelines for HIV Testing 2008. London: BHIVA 2008.

8 Branson BM, Handsfield HH, Lampe MA et al. Revised recommendations for HIV testing of adults, adolescents, and pregnant women in health-care settings. MMWR Recomm Rep 2006;55:1-17. 
9 WHO / UNAIDS. Policy Statement on HIV Testing, June 2004.

www.who.int/ethics/topics/en/hivtestingpolicy_who_unaids_en_2004.pdf (accessed Jan 2011).

10 Rogstad K, Palfreeman A, Rooney G, et al. UK national guidelines on HIV testing 2006. Int J STD AIDS 2006;17:668-676.

11 UNAIDS. Intensifying HIV prevention : a UNAIDS policy position paper. Geneva: UNAIDS 2005.

12 UNAIDS. Practical guidelines for intensifying HIV prevention: Towards universal access. Geneva: UNAIDS 2007.

13 Vidanapathirana J, Abramson MJ, Forbes A, et al. Mass media interventions for promoting HIV testing. Cochrane Database Syst Rev 2005;3 : CD004775.

14 Rees R, Kavanagh J, Burchett H, et al. HIV Health Promotion and Men who have Sex with Men (MSM): A systematic review of research relevant to the development and implementation of effective and appropriate interventions. London: EPPI-Centre 2004.

15 Lorenc T, Marrero-Guillamón I, Llewellyn A, et al. Preventing and reducing HIV transmission among men who have sex with men (MSM): Increasing the uptake of HIV testing. Systematic review of effectiveness, cost-effectiveness and qualitative evidence. London: National Institute for Health and Clinical Excellence 2010. 16 National Institute for Health and Clinical Excellence (NICE). Methods for the development of NICE public health guidance (second edition). London: National Institute for Health and Clinical Excellence 2009.

17 Galvan FH, Bluthenthal RN, Ani C, et al. Increasing HIV testing among Latinos by bundling HIV testing with other tests. J Urban Health 2006;83:849-859. 
18 Spielberg F, Branson BM, Goldbaum GM, et al. Choosing HIV counseling and testing strategies for outreach settings: a randomized trial. J Acquir Immune Defic Syndr 2005;38:348-55.

19 Spielberg F, Critchlow C, Vittinghoff E, et al. Home collection for frequent HIV testing: acceptability of oral fluids, dried blood spots and telephone results. AIDS 2000;14:1819-1827.

20 Wilton L, Herbst JH, Coury-Doniger P, et al. Efficacy of an HIV/STI prevention intervention for black men who have sex with men: findings from the Many Men, Many Voices (3MV) Project. AIDS Behav 2009;13:532-44.

21 Flowers P, Hart GJ, Williamson LM, et al. Does bar-based, peer-led sexual health promotion have a community-level effect amongst gay men in Scotland? Int J STD AIDS 2002;13:102-8.

22 Elford J, Bolding G, Sherr L. Peer education has no significant impact on HIV risk behaviours among gay men in London. AIDS 2001;15:535-8.

23 Golden MR, Gift TL, Brewer DD, et al.Peer referral for HIV case-finding among men who have sex with men. AIDS 2006;20:1961-8.

24 Guy R, Goller J, Leslie D, et al. No increase in HIV or sexually transmissible infection testing following a social marketing campaign among men who have sex with men. J Epidemiol Community Health 2009;63:391-6.

25 McOwan A, Gilleece Y, Chislett L, et al. Can targeted HIV testing campaigns alter health-seeking behaviour? AIDS Care 2002;14:385-90.

26 Chiasson MA, Shaw FS, Humberstone M, et al. Increased HIV disclosure three months after an online video intervention for men who have sex with men (MSM). AIDS Care 2009;21:1081-9. 
27 Dukers-Muijrers NH, Niekamp AM, Vergoossen MM, et al. Effectiveness of an opting-out strategy for HIV testing: evaluation of 4 years of standard HIV testing in a STI clinic. Sex Transm Infect 2009;85:226-30.

28 Heijman RL, Stolte IG, Thiesbrummel HF, et al. Opting out increases HIV testing in a large sexually transmitted infections outpatient clinic. Sex Transm Infect 2009;85:249-55.

29 Ryder N, Bourne C, Rohrsheim R. Clinical audit: adherence to sexually transmitted infection screening guidelines for men who have sex with men. Int J STD AIDS 2005;16:446-9.

30 Flowers P, Frankis JS, Hart GJ. Evidence and the evaluation of a community-level intervention: researching the Gay Men's Task Force Initiative. In: Watson J, Platt S, eds. Researching health promotion. London: Routledge 2000: 102-124.

31 Elford J, Sherr L, Bolding G, et al., 2000. Peer-led HIV prevention among gay men in London (the 4 gym project). Intervention and evaluation. In: Watson J, Platt S, eds. Researching health promotion. London: Routledge 2000: 207-230.

32 Wakefield MA, Loken B, Hornik CR. Use of mass media campaigns to change health behaviour. Lancet 2010;376:1261-71.

33 Eaton LA, Kalichman SC, Cain DN, et al. Serosorting sexual partners and risk for HIV among men who have sex with men. Am J Prev Med 2007;33:479-85.

34 Hart GJ, Elford J. Sexual risk behaviour of men who have sex with men: emerging patterns and new challenges. Curr Opin Infect Dis 2010;23:39-44. 
Figure 1. Flow of literature through the review

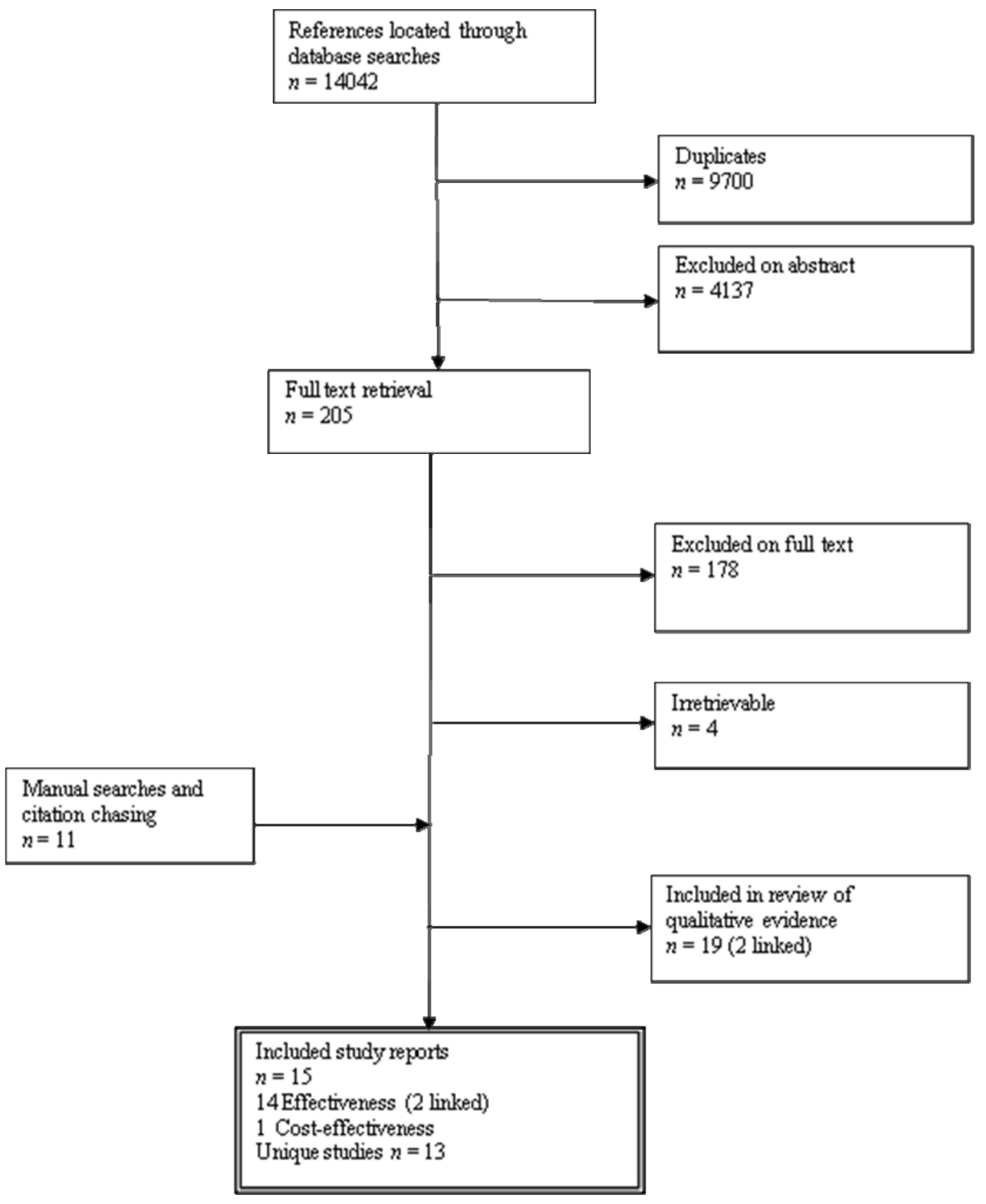


Supplement 1. Search strategy used for the Medline database

1. gay and (men or male\$).mp

2. Homosexuality, Male/ or homosexual\$.mp

3. exp homosexuality/

4. Men who have sex with men.mp

5. same sex.mp

6. MSM.mp

7. (male and (sex work $\$$ or prostitute\$ or transactional sex $\$$ )).mp

8. bisexual.mp or bisexuality/

9. or $/ 1-8$

10. exp hiv/

11. exp hiv-1/

12. exp hiv-2/

13. Human immunodeficiency virus.mp

14. hiv.mp

15. or/10-14

16. test\$.mp

17. VCT.mp

18. voluntary counselling and testing.mp or voluntary counselling and testing.mp

19. PIT.mp

20. Provider initiated testing.mp

21. client initiated testing.mp

22. (sexual health or gum or sti and (service $\$$ or clinic)).mp

23. or/16-22

24. (attend\$ or non-attend $\$$ or increas $\$$ or promot\$ or opt $\$$ or particip\$ or adherence or involvement or uptake or take-up or utilize or utilise or refus\$ or referr\$ or self-referr\$ or barrier\$ or decrease \$ or interven\$ or aware\$ or opportunit\$).mp

25. Attitude to health/

26. Health service accessibility.mp

27. Access to information/

28. Health education/

29. Health promotion/

30. Preventive health services/

31. Sexual behaviour.mp

32. Patient acceptance of health care/

33. Patient compliance/

34. Risk reduction behavior/ or Risk reduction behaviour/

35. Risk-taking/

36. Motivation/

37. Stigma.mp

38. (health $\$$ adj3 (educat $\$$ or aware $\$$ or opportunit $\$$ or attitude $\$$ or access $\$$ or inform $\$$ or promot $\$$ or prevent $\$$ or behavio?r\$)).ti,ab.

39. (sex $\$$ adj2 (behavio? $\$$ or educat $\$)$ ).ti,ab.

40. (risk\$ adj3 (taking or factor\$ or behavio?r\$ or educat\$ or reduc\$)).ti,ab.

41. (patient $\$$ adj3 (satisfaction or educat\$ or behavio? $\$$ or compliance or comply or complie $\$))$.ti,ab.

42. (barrier\$ or facilitat $\$$ or hinder $\$$ or block $\$$ or obstacle $\$$ or restrict $\$$ or restrain $\$$ or obstruct\$ or inhibit\$ or impede $\$$ or delay $\$$ or constrain $\$$ or hindrance).ti,ab. 
43. (attitude $\$$ or opinion $\$$ or belief $\$$ or perceiv $\$$ or perception $\$$ or aware $\$$ or personal view $\$$ or motivat $\$$ or incentive $\$$ or reason\$).ti,ab.

44. Or/25-43

45. 24 OR 44

46. 9 and 15 and 23 and 45

47. limit 46 to (English language and humans)

48. limit $47 \mathrm{yr}=$ " $1996-$ Current" 
Supplement 2: data extraction and quality assessment tools

\section{Quality assessment: effectiveness studies}

Questions:

1. Is the source population or source area well described?

2. Is the eligible population or area representative of the source population or area?

3. Do the selected participants or areas represent the eligible population?

4. How was confounding minimised?

5. Were interventions (and comparisons) well described and appropriate?

6. Was the allocation concealed?

7. Were participants and/or investigators blind to exposure and comparison?

8. Was the exposure to the intervention and comparison adequate?

9. Was contamination acceptably low?

10. Were other interventions similar in both groups?

11. Were all participants accounted for at study conclusion?

12. Did the setting reflect usual UK practice?

13. Did the intervention or control comparison reflect usual UK practice?

14. Were the outcome measures reliable?

15. Were all outcome measurements complete?

16. Were all important outcomes assessed?

17. Were outcomes relevant?

18. Were there similar follow-up times in exposure and comparison groups?

19. Was follow-up time meaningful?

20. Were exposure and comparison groups similar at baseline? If not, were these adjusted?

21. Was Intention to Treat (ITT) analysis conducted?

22. Was the study sufficiently powered to detect an intervention effect (if one exists)?

23. Were the estimates of effect size given or calculable?

24. Were the analytical methods appropriate?

25. Was the precision of intervention effects given or calculable? Were they meaningful?

26. Are the study results internally valid? (i.e. unbiased)

27. Are the study results generalisable to the source population? (i.e. externally valid)

Answers 1-25:

++ The study has been designed/conducted in such a way as to minimise the risk of bias

+ Either the answer to the checklist question is not clear from the way the study is reported, or the study may not have addressed all potential sources of bias

- Significant sources of bias may persist

NR The study fails to report this particular question

NA Not applicable given the study design

Answers 26-27:

++ All or most of the checklist criteria have been fulfilled; where they have not been, the conclusions are very unlikely to alter 
+ Some of the checklist criteria have been fulfilled, where they have not, or not adequately described, the conclusions are unlikely to alter

- Few or no checklist criteria have been fulfilled and the conclusions are likely to alter

NB. The answer to question 26 (internal validity) is the overall QA score given to each reference in the main text of the paper.

\section{Quality assessment: cost-effectiveness studies}

Questions:

1. Is the study population appropriate for the topic being evaluated?

2. Are the interventions appropriate for the topic being evaluated?

3. Is the system in which the study was conducted sufficiently similar to the UK context?

4. Were the perspectives clearly stated?

5. Are all direct health effects on individuals included, and are all other effects included where they are material?

6. Are all future costs and outcomes discounted appropriately?

7. Is the value of health effects expressed in terms of quality adjusted life years (QALYs)?

8. Are costs and outcomes from other sectors fully and appropriately measured and valued?

9. Overall judgment (no need to continue if not applicable)

10. Does the model structure adequately reflect the nature of the topic under evaluation?

11. Is the time horizon sufficiently long to reflect all important differences in costs and outcomes?

12. Are all important and relevant outcomes included?

13. Are the estimates of baseline outcomes from the best available source?

14. Are the estimates of relative "treatment" effects from the best available source?

15. Are all important and relevant costs included?

16. Are the estimates of resource use from the best available source?

17. Are the unit costs of resources from the best available source?

18. Is an appropriate incremental analysis presented or can it be calculated from the data?

19. Are all important parameters whose values are uncertain subjected to appropriate sensitivity analysis?

20. Is there any potential conflict of interest?

21. Overall assessment

Answers to questions 1-20:

Yes; Partly; No; Unclear; Not Applicable

\section{Data extraction: effectiveness studies}

Author:

Year:

Citation:

Aim of study:

Study design: 
Source population/s:

Eligible population:

Selected population:

Excluded population/s:

Setting:

Method of allocation:

Intervention/s description:

Control/comparison/s description:

Sample sizes: [total; intervention; control]

Baseline comparisons:

Study sufficiently powered?

Primary outcomes:

Secondary outcomes:

Follow-up periods:

Method of analysis:

Results - primary outcomes:

Results - secondary outcomes:

Attrition details:

Limitations identified by author:

Limitations identified by review team:

Evidence gaps and/or recommendations for future research:

Source of funding:

Data extraction: cost-effectiveness studies

Author:

Year:

Citation:

Type of economic analysis:

Economic perspective:

Setting:

Data sources:

Intervention/s description:

Control/comparison/s description:

Sample sizes: [total; intervention; control]

Primary outcomes:

Secondary outcomes:

Time horizon:

Discount rates:

Modelling method:

Results - primary analysis:

Results - secondary analysis:

Limitations identified by author:

Limitations identified by review team:

Evidence gaps and/or recommendations for future research:

Source of funding: 